\title{
Sich schreiben im Schatten des Todes
}

\author{
Zu Sandra Butlers und Barbara Rosenblums Cancer in two \\ voices (1991)
}

Simon Peng-Keller

In der Fülle an publizierten Lebensenderzählungen nimmt Sandra Butlers und Barbara Rosenblums Buch Cancer in two voices eine Sonderstellung ein. ${ }^{1}$ Wie schon der Titel verrät, wird hier aus zwei Perspektiven erzählt: Auf der einen Seite steht Barbara Rosenblum, die an Krebs erkrankt und schließlich stirbt, auf der anderen Seite Sandra Butler, die ihre Lebenspartnerin in Krankheit und Sterben begleitet und in Trauer zurückbleibt. Es ist der Wechsel zwischen diesen beiden Perspektiven, der die Lektüre dieses Buches in besonderer Weise bestimmt. Die Lesenden werden hineingenommen in ein anrührendes Lebensschicksal und bewegen sich in fortwährendem Wechsel zwischen zwei Perspektiven hin und her - bis am Ende nur noch die Perspektive Butlers bleibt, die den Tod ihrer Geliebten und die Trauer um sie beschreibt. Das Buch, das aus diesem wechselseitigen Sich-Schreiben (im mehrfachen Sinne dieses Wortes) entsteht, ist nicht in erster Linie ein Buch über Krankheit und Tod, sondern in viel stärkerem Maße ein Zeugnis, was es bedeutet, "angesichts des unausweichlichen Todes intensiv, offenherzig und freudvoll zu leben« (5). Wer sich auf dieses zweistimmige Erzählen einlässt, erlebt lesend die wachsende Todesnähe mit. Lesezeit ist Lebenszeit. Mit den gelesenen Seiten zerrinnt die Zeit, die noch bleibt. Gegen Ende werden Rosenblums Einträge kürzer, seltener, bis ihre Stimme ganz verstummt. Sie stirbt 1988 im Alter von 44 Jahren.

Der vorliegende Beitrag entstand im Rahmen des Forschungsprojekts "Sterbenarrative«, das untersucht, in welcher Weise gegenwärtig am und vom Lebensende erzählt wird und was der Sinn solchen Erzählens darstellt. Butlers und Rosenblums Buch ist in diesem $\mathrm{Zu}-$ sammenhang allein schon deshalb so anregend, weil es selbst, wie im ersten Abschnitt gezeigt werden soll, auf die Erfahrungen reflektiert, die mit dem Schreiben am Lebensende verknüpft sind. Die darauf folgenden Abschnitte zeichnen die vier Storylines nach, die dem Buch eine dichte narrative Struktur verleihen. Dass ich hier von

\footnotetext{
1 Sandra Butler/Barbara Rosenblum, Cancer in two voices, London 1994. Die Zitate wurden von mir übersetzt.
} 
"Storylines" spreche und nicht, was vielleicht naheliegender wäre, von Handlungssträngen oder Plots, ist in der Sache selbst begründet: Im Unterschied zum deutschen Wort "Handlungsstrang", das einseitig die aktive Dimension des Erzählten betont, und dem narrativen Terminus des »Plots«, der für faktuales Erzählen zu artifiziell wirkt, hat das elegante Fremdwort "Storyline« den Vorzug größerer Offenheit für lebensgeschichtliche Passivitäten und eine metaphorische Nähe zum chiromantischen Terminus der "Lebenslinie«, an der sich angeblich die Schwankungen und Ereignisse eines Lebenslaufs abzeichnen.

\section{»Ein Spiegel, der die eigenen Entscheidungen klärt ...« Vom Sinn des Erzählens am und vom Lebensende}

Rosenblums und Butlers Zeugnisbericht entsteht aus einem sich während drei Jahren entfaltenden Spiegelungs- und Resonanzgeschehen, einem fortlaufenden Bezeugen eigener Erfahrungen im gleichzeitigen Hören auf das, was die Partnerin erfährt und erzählt. Dass das Aufschreiben des Erlebten zunächst der Selbstklärung dient, wird mehrfach betont. Dennoch wird das Schreiben auch von der Absicht geleitet, ein Buch zu verfassen, das für andere in einer ähnlichen Situation hilfreich sein könnte. In ihrer Einleitung schreibt Butler, kurz nach der Diagnose sei der gemeinsame Entschluss gefallen, ein Buch zu schreiben - »als eine Landkarte unserer Erfahrungen, in der Hoffnung, dass dies für Euch, die ihr es nun lest, nützlich sei« (3). Als Anstoss und Modell diente Barbara Myerhoffs Film In Her Own Time, in dem die ebenfalls an Krebs erkrankte USamerikanische Ethnologin die Vorbereitung auf ihr Sterben filmisch dokumentierte. Den Abend, an dem sie sich diesen Film anschaute, bezeichnet Rosenblum als einen »Wendepunkt ihres Lebens" (51): »[M] eine kreativen Säfte waren so elektrisch wie nie zuvor; meine Gedanken waren noch nie so klar." (56) An diesem Abend fand sie die Klarheit über den Weg, der vor ihr lag und zu dem auch die schriftliche Bezeugung ihrer eigenen Erfahrungen gehörte. Myerhoffs Film vermittelte ihr einen Sinn für die therapeutische Kraft, die dem Erzählen am und vom Lebensende innewohnt: "Zu sehen, wie andere ihre letzte Monate auf Erden verbringen, kann ein Spiegel darstellen, um die eigenen Entscheidungen zu klären." (51)

Rosenblum hatte das Kino in der Erwartung betreten, von Myerhoff konkrete Hinweise für ihre eigene Suche zu bekommen. Sie 
teilte mit ihr nicht nur Vorname und Krankheit, sondern auch ihren Beruf und ihre jüdische Herkunft. Während des Films wird ihr jedoch bewusst, dass sie ihren eigenen Weg finden muss, sich vom Leben zu verabschieden. Bemerkenswerterweise war es eine Kontrasterfahrung, die Rosenblum die Augen öffnete. Myerhoff gab ihr zwar ein Modell für ihren eigenen Bericht vor, doch keines für den Umgang mit ihrem Schicksal. Während Myerhoff bis zuletzt in der Rolle der beobachtenden Ethnologin bleibt und diese dazu benutzt, Krankheit und Tod auf Distanz zu halten, entschließt sich Rosenblum, das verbleibende Leben in allen Fasern auszukosten und die verbleibende Kraft dazu zu nutzen, neu zu erkunden, was sie bisher vernachlässigt hatte: »Ich wollte singen und tanzen, Musik machen und reisen." (54) In ihren Notizen gibt sie Anteil an der neuen und letzten Lebensreise, die an diesem Abend begann.

Cancer in two voices verbindet verschiedene Formen autobiografischen Erzählens. Zum größeren Teil besteht das Buch aus datierten Tagebucheinträgen, die die Zeitspanne zwischen dem 19. Februar 1985 und dem 14. Februar 1991 umfassen. Diese Einträge sind, entsprechend zu Lebens- und Krankheitsphasen, in zwölf Kapitel unterteilt, in deren Überschrift sich jeweils ein Leitthema ankündigt. Rosenblums Rundbriefe, die den Freundeskreis über das Fortschreiten der Krankheit informieren, gleichen Meilensteinen im Erzählfluss. Die Abfolge der Tagebuchnotizen wird unterbrochen durch sieben Zwischenreflexionen, die je einem besonderen Thema gewidmet sind. Hinweise auf den gemeinsamen Schreibprozess tauchen an verschiedenen Stellen auf. Die Chronologie der Abfolge wird gleich zu Beginn schon durchbrochen. Sandra Butler nimmt in ihrer Einleitung das Datum, welches das Erzählen bestimmt, gleich vorweg: "Am 14. Februar 1988, dem Valentinstag, starb Barbara Rosenblum. Sie war meine Lebenspartnerin, meine Freundin und meine Geliebte." (3) Ihr ist das Buch auch gewidmet: „Für Barbara: zichrona l'bracha - gesegnet sei das Gedächtnis an sie." Die Widmung macht das gemeinsame Vermächtnis zu einer Gedenkschrift für die Verstorbene.

\section{Storyline 1: Fehldiagnose und »Blutgeld»}

Die erste Handlungs- und Leidenslinie, welche die erste und letzte Peripetie des Buches bestimmt, bildet die Krankheit selbst. Sie steht von Anfang an in einem komplizierten Verhältnis zur kurativen Medizin. Wenige Monate nachdem ein Brustkrebs in einem bereits 
fortgeschrittenen Stadium entdeckt worden war, bestätigte ein Fremdgutachten die Vermutung, dass der Radiologe, der zu einem viel früheren Zeitpunkt eine Mammografie durchführte, das bereits damals sichtbare Geschwür übersehen hatte. Auf den Schock der Diagnose folgt der Schock der Einsicht, dass aufgrund ärztlichen Versagens die Therapie vielleicht zu spät einsetzte: „Ich weiß, dass Menschen fortwährend Fehler machen. [...] Doch dieser Fehler betrifft mein Leben.«(29) Rosenblum entdeckt schrittweise den institutionellen Hintergrund dieses ärztlichen Versagens - und die biografischen Gründe, weshalb sie selbst die Logik der Zweiklassenmedizin nicht schon viel früher durchschauen konnte. Als Tochter jüdischer Eltern, die vor dem 2. Weltkrieg mit wenig finanziellen Mitteln eingewandert waren und sich zeitlebens mit der USamerikanischen Geschäftswelt schwertaten, hatte sie nicht gelernt, zwischen unterschiedlichen medizinischen Behandlungsqualitäten zu unterscheiden und um eine gute Versorgung zu kämpfen: »Ich hatte mich an eine schludrige Medizin gewöhnt und glaubte, dass die eilige Massenmedizin, die ich bekam, dem medizinischen Standard entsprach.«(30)

Sucht man in der Krankheits- und Sterbegeschichte Rosenblums, die sich während der Lektüre aus den Erzählfragmenten ihres Tagebuchs zusammensetzt, nach einem tragischen Plot, so besteht er in dieser Verwicklung, in der sich eine biografisch-familiäre Belastung mit einem systembedingten Versagen kreuzt und in eine persönliche Katastrophe mündet. Dass Rosenblum gegen das verantwortliche Krankenhaus einen Rechtsprozess anstrengt und schließlich auch gewinnt, bringt nicht nur Entlastung, sondern erzeugt auch neue Ambivalenzen. Die Schadenersatzzahlung von 296'000 Dollar befreit sie zwar von finanziellen Sorgen. Doch stört sie die bisherige Beziehungssymmetrie zwischen ihr und ihrer Partnerin und verwickelt Rosenblum in eine Auseinandersetzung um eine gerechte Erbverteilung. Das »Blutgeld« (89) bedarf der Erlösung.

\section{Storyline 2: Liebe im Zeichen des Abschieds}

In die Lebens- und Liebesgeschichte der beiden Autorinnen werden die Leserinnen und Leser durch die schon zitierte Einleitung eingeführt, die Sandra Butler nach dem Tod ihrer Freundin schrieb:

»Barbara und ich trafen uns im Jahre 1979. Sie war damals Assistenzprofessorin in Stanford, die gerade eine Festanstellung abgelehnt hatte und daran war, eine wichtige Beziehung zu 
beenden. Ich selbst war vor kurzem von San Francisco nach Paolo Alto umgezogen [...]. Wir beide waren städtische Frauen, die in den letzten zwei Jahrzehnten in New York, Chicago, San Francisco und Boston gelebt hatten. Beide hatten wir als Heterosexuelle gelebt, bevor wir in den vergangen zehn Jahren vor unserer Begegnung zunehmend Liebesbeziehungen mit Frauen eingegangen waren. Beide waren wir Jüdinnen - sie aus einer fortschrittlichen Einwandererfamilie, die erst seit einer Generation in den USA waren, während ich einer mittelständischen, assimilierten, vorstädtischen Familie entstammte.« (4)

Das zweistimmige Erzählen entfaltet eine besondere Kraft, wenn es um die Vergegenwärtigung der Liebesgeschichte geht, die 1979 während einer Buchvernissage begann. "Worte waren unsere Währung, unser Medium", schreibt Sandra Butler im Rückblick auf die gemeinsamen Jahre (3). Der Entscheid, gemeinsam ein Buch zu schreiben, lag nahe. Zum bereits genannten Motiv, anderen in einer ähnlichen Situation etwas an die Hand zu geben, fügte sich ein zweites hinzu: Das Schreiben sollte dazu dienen, das, was sie durchlebten, für sich selbst sichtbar zu machen (3). Das gemeinsame Schreiben ermöglichte, sich zu erkunden und zu erschließen. »Das Erzählen schafft Nähe." (49) Es verdichtete und klärte die Beziehung. Und es ermöglichte eine Intimität, die Butler an die Zeit vor dem Ausbruch der Krankheit erinnerte:

"Die Arbeit, die wir vollbrachten, hatte jene Fokussierung, jene Leidenschaft, jenen Sinn für Vollendung, die unsere sexuellen Begegnungen einst hatten. Häufig fühlte ich mich ähnlich erschöpft, wenn eine Arbeitssitzung endete. Ebenso geliebt. Ebenso erkannt. So tief verbunden mit dieser Frau.« (141)

Rosenblum selbst beschreibt die beziehungsstiftende Qualität des gemeinsamen Schreibens in einem Abschnitt, in dem sie über den krankheits- und therapiebedingten Rückgang ihres sexuellen Begehrens reflektiert:

"Zuletzt bleibt die grundlegende Frage: Wenn jemand mit dem Tod konfrontiert ist, was geschieht dann mit der eigenen Sexualität? Ich vermute, dass bei manchen Frauen sich die sexuellen Gefühle intensivieren. Sie entwickeln Lebenshunger, Lebenshunger durch Sexualität. Erotische Energie hält sie am Leben. Ich vermute, Sandy hätte es lieber gehabt, wenn ich die Lebensenergie als erotische Energie erleben würde, als Libido. Aber ich erlebe es anders. Meine Lebensenergie zeigt sich in 
einer anderen Form, in der Leidenschaft, alles zu lernen, alles zu fühlen, jeden Moment mit Präsenz und Intensität zu erleben. Neue Dinge zu studieren. Neue Wissensgebiete zu beherrschen. $\mathrm{Zu}$ schreiben - allein und mit Sandy. Zusammen haben wir eine neue Form entwickelt, die unsere individuellen und einzigartigen Stimmen in einem Dialog aufeinander abstimmen kann. Wir schreiben über Dinge, die wichtig für uns sind. Wir schlafen miteinander vor der Schreibmaschine, nicht im Schlafzimmer.» (132)

Die Spannungen, die schon die ersten gemeinsamen Jahre prägten, verstärken sich zeitweise durch die Krankheit. Der Krebs verändert das gemeinsame Leben in jederlei Hinsicht und machtVerletzlichkeit und Abhängigkeit spürbarer. Als der Tod absehbar wird, stellt sich schließlich die Einsicht ein, dass nicht mehr genügend Zeit bleiben wird, um alle Beziehungsfragen noch gemeinsam durchzuarbeiten. Die Zeit der Beziehungskämpfe kommt an ihr Ende: "Statt dessen gilt unser Kampf nun dem Sinn, der Bewahrung derVerbundenheit, dem Bewusstsein. « (73)

Um die Spannung zwischen Verbundenheit und Abschied bewusst zu gestalten, entscheidet das Paar, sich im Kreise der Freundinnen trauen zu lassen. Die Suche nach der passenden Form stärkt die Beziehung und weckt Kreativität. In der Zeremonie mischen und verbinden sich verschiedene Traditionen. Ein tibetanischer Gong dient zur Eröffnung. Danach spricht eine Freundin ein jüdisches Segensgebet. Es folgen kurze, bewegende Ansprachen, in denen der Dank für den bisherigen gemeinsamen Weg und das Versprechen gegenseitiger Liebe zum Ausdruck kommt. Segenswünsche und Gebete bereiten schließlich die Ringübergabe vor. In Musik und ausgelassenem Tanz klingt der Abend aus. "Heute war der glücklichste Tag meines Lebens", sind Barbara Rosenblums letzte Worte vor dem Einschlafen (100).

Die letzten Lebensmonate stehen im Zeichen des Abschieds und der Trauer, die beide Frauen auf je eigene Weise bewältigen. Phasen intensiverVerbundenheit und solche des Rückzugs wechseln sich ab. Butler kommt die Aufgabe zu, den Freundeskreis über das herannahende Ende zu informieren und Rosenblums Familie in den Abschiedsprozess einzubeziehen. Die Öffnung der intimen Zweisamkeit, das schrittweise Zurücktreten, um auch anderen Raum für den Abschied zu lassen, bedeutet ein weiteres Stück Trauerarbeit. Sie notiert: 
»Ab jetzt werden immer andere um uns sein. Ich muss den Erstanspruch auf unsere gemeinsame Zeit zurücklassen, eine weniger zentrale Rolle einnehmen, ihr bei ihrem Abschied beistehen, ihr helfen, ihre Arbeit hier zu beenden. Das ist jetzt die Form meines Respektes und der Freundschaft. Mein einsamer Ausdruck der Liebe." (152)

Ein gemeinsames Projekt bleibt ihnen: das Buch, an dem sie fortwährend schreiben und das den Raum dafür schafft, über die innersten Gefühle und Nöte ins Gespräch zu kommen (158). Zwei Wochen vor ihrem Tod schreibt Rosenblum einen letzten Rundbrief, um sich von ihren Freundinnen zu verabschieden. Darin heißt es: "Sandy und ich sind daran, ein Buch zu vollenden, dass die Reise meines Krebses nachzeichnet. Es trägt den Titel: Cancer in two voices.« (166) Das letzte Liebesbekenntnis murmelt Butler am Bett ihrer eben verstorbenen Geliebten: »Ich liebe dich. Gehe behutsam dem Licht entgegen, meine Liebste. Geh mit Liebe und mit Ruhe. S'hmaYisrael Adonai Elohenu Adonai Echod. Geh in Frieden dem Licht entgegen, meine Liebste.«(170)

\section{Storyline 3: Zurück zu den jüdischen Wurzeln}

Das Buch, das Barbara Rosenblum und Sandra Butler gemeinsam schreiben, erzählt auch, auf welchen Wegen die beiden Autorinnen im Verlauf der erzählten Zeit stärker zu ihren jüdischen Wurzeln zurückfinden. Aus der religiösen Innenperspektive kann das Buch als ein Heimkehrbericht gelesen werden. Die religiöse Herkunft ist durch die bereits zitierte Eröffnung von Anfang an präsent und tritt im Laufe des Buches zunehmend in den Vordergrund. In den Tagebuchnotizen der ersten Wochen nach der Diagnose findet sich das Thema nur vereinzelt. Nach der Erholung von der ersten Chemotherapie, als die Haare wieder zu wachsen beginnen, erlebt Rosenblum einen Augenblick des Glücks und notiert in ihr Tagebuch, dass sie sich nun wieder weniger verletzlich fühle und ihr Kopf von Gottes Gegenwart und des Lebens bedeckt sei (28). Am Yom Kippur desselben Jahres besuchen die beiden zusammen die Synagoge und schluchzen während der ganzen Feier (44). Das beschriebene Trauungsritual mit vielen jüdischen Elementen bedeutet ein weiterer Schritt, sich in die religiöse Tradition ihrer Vorfahren zu stellen. Im April 1987 reist das Paar nach New York, um zusammen mit Rosenblums Eltern ein letztes Mal gemeinsam Geburtstag zu feiern. Rosenblum schreibt: "Ich wurde am 9. April 1943 geboren, zehn 
Tage vor dem Aufstand [im Warschauer Ghetto]. Sandy wurde am 19. April 1938 geboren, fünf Jahre früher, aber am selben Datum.» (114) Rosenblum erinnert sich, dass ihre Eltern am Tage des Warschauer Aufstands seit jeher zu einem Gedenkanlass gehen, während sie selbst bisher davor zurückschreckte - bis zum 44. Gedenktag, der auf ihren 44. Geburtstag folgte. Sandra Butler beschreibt diesen denkwürdigen Tag:

"Ich saß da und hörte auf den tröstlichen, vertrauten, unentzifferbaren Klang des Jiddischen und begann zu weinen. Ich ließ die Tränen mein Gesicht herunterrinnen und in meinen Mund tropfen. Doch als ich herumschaute, sah ich, dass ich die einzige war, die weinte. Denn diese betagten Menschen hatten während vieler Jahre und Jahrzehnte geweint. [...] Ich begann, mich mit meinem Weinen anmaßend zu fühlen. Weinen war zu einfach. In diesen schwerfalligen Körpern und unerschrockenen Gesichtern spielte sich etwas viel Komplizierteres ab. Etwas, das ich noch nicht verstand. Sich ein Verstehen vorzustellen, das ein solches Leiden, ein solcher Verlust umfasste, schien unverschämt zu sein. Barbara würde sterben, wohl innerhalb eines Jahres. Wir wussten es beide. Ich war daran, mich auf eine Erfahrung vorzubereiten, die der tiefgreifendste Verlust meiner fast fünfzig Jahre werden würde. [...] Aber all diese Leute in diesem kleinen Elementarschulzimmer hatten Kinder verloren, Männer, Frauen, Eltern, Freunde, Tanten, Nachbarn. Und sie hatten weitergemacht.Vielleicht ist das schlichte Aushalten der Weg, ein Zeugnis abzulegen." (117)

In den Wochen vor ihrem Tod sucht Rosenblum den Kontakt zu einem ihr nahestehenden Rabbiner. Sie trifft sich mehrfach mit ihm, um ihre jüdische Identität weiter zu klären und eine angemessene rituelle Form der Sterbevorbereitung zu finden. Nach intensiver Lektüre entscheidet sie sich schließlich zu einer speziellen Mikwe, einem rituellen Taufbad, um sich auf den letzten Übergang, ihren letzten Sabbat, vorzubereiten. In ihrem letzten Brief an ihre Freundinnen schreibt sie:

"Ich fühle mich so, als sei ich in eine heilige Zeitspanne eingetreten, in der es kein Wissen mehr gibt, sondern allein Glaube und Hoffnung. [...] Kürzlich habe ich mich auch auf eine transformative Erfahrung eingelassen, die als Mikwe bekannt ist und aus einem rituellen Taufbad besteht. Gewöhnlich wird es dazu benutzt, um einen Zustand der Heiligkeit oder der Vereinigung mit Gott einzutreten. Ich habe entdeckt, dass ich eine Frau mit 
tiefem Glauben bin, und ich bin bewegt von den [jüdischen] Lehren. Eine Freundin mit einem quäkerischen Hintergrund sagte mir, sie halte mich im Licht. Ein anderer Freund, ein ehemaliger Priester, sagte mir, dass wir uns an einem besseren Ort wiederbegegnen werden. All diese Bilder und Metapher sprechen mich an, und ich sehe eine Gemeinsamkeit zwischen ihnen. Am meisten sprechen mich jedoch spezifisch jüdische Bilder und kulturelle Themen an: Musik, Essen, Sprache und mein religiöses Erbe.«(163)

Zusammen mit Rabbi Kahn bereitet Rosenblum zwei Wochen vor ihrem Tod ihre Abdankungsfeier und ihr Begräbnis vor. Mit Blick auf ihre Eltern entscheidet sie sich für das traditionelle jüdische Ritual. Sie bittet Rabbi Kahn ihren Vermächtnisbrief an den einzigen Neffen vorzulesen, der noch zu klein ist, um ihn zu verstehen. Nicht nur die Abdankungsfeier vollzieht sich in jüdischer Tradition, auch für die Trauerzeit wählt Butler eine traditionell jüdische Form. An einem Punkt allerdings wird die Traditionstreue, dem letzten Willen Rosenblums folgend, für eine alternative Form geöffnet. Genau ein Jahr nach der oben beschriebenen Traufeier versammeln sich dieselben Freundinnen nochmals, diesmal um Abschied zu nehmen:

»Als die Sonne am Freitag, dem 19. Februar unterging, versammelten sich alle, die zur Feier des letzten Sabbats eingeladen waren, im Wohnzimmer. [...] Ich stand am Glastisch, als die Kerzen angezündet und die Gebete zum Sabbat rezitiert wurden. Mit brennenden Kerzen versammelten wir uns in Barbaras Garten. Jane ließ dieselben Tibetanischen Glocken erklingen, die unsere Traufeier eröffnet hatten.«(173)

Zur Feier haben alle eine Pflanze mitgebracht. Bevor die Asche im ganzen Garten verstreut wird, knien alle nieder, um etwas einzupflanzen - »im Gedenken an Barbaras Leben, Geist und Liebe für das Wachsende« (174).

\section{Storyline 4: »Mein Sterben« - »Barbara stirbt«}

Im Unterschied zum Leser, zur Leserin, wissen die beiden Autorinnen zu Beginn nicht, wie lange die "Reise mit dem Krebs« dauern wird. Die Ärzte prognostizierten eine Überlebenszeit von fünf bis acht Jahren. Am Ende sind es drei. Zunächst wird alles medizinisch Mögliche versucht, um den Krebs zu entfernen: Mastektomie, Bestrahlung, Chemotherapie. Die Einsicht, dass die Krankheit Ro- 
senblums Leben stark verkürzen würde, ist von Anfang an da. Drei Monate nach der Diagnose schreibt Butler: \#Barbara wird sterben. Es ist nun nur noch eine Frage der Jahre. Ich wiederhole diese neue Realität wieder und wieder, ähnlich wie ich es mit der Diagnose am Anfang tat." (26) Etwa einen Monat später braucht auch Rosenblum erstmals die Formel »in my dying« (29). Und wenige Tage später:»Heute fühle ich mein Todesurteil schmerzlich. [...] Die Zeit schrumpft mehr und mehr, sie zieht sich zurück wie der Kopf einer Schildkröte.« (32) Die Tagebuchnotizen zeichnen die oszillierende Linie zwischen Optimismus und Verzweiflung nach, die durch die Mitteilung der neuen Befunde hart getaktet wird. Auf Phasen der Entspannung folgen solche der Verzweiflung und der Trauer, dass sich die Therapien als weniger wirksam erweisen, als zu erhoffen war. Die Tage gewinnen an Reichtum und emotionaler Spannbreite: »Ich bin erfüllt mit einem Gefühl, da zu sein, bewegt zu sein, berührt $\mathrm{zu}$ sein und ebenso verbunden wie allein in meinem Leben, meiner Reise, meiner Odyssee." (36)

Es sind Abschiedsreisen, die Rosenblum bereits im ersten Jahre ihrer Krankheit beginnt. Butler begleitet und unterstützt sie. Abschiedsreisen sind es auch für sie. Sie führen an Orte, die Rosenblum in ihrem Leben wichtig waren, und an solche, die sie immer schon sehen wollte. Zugleich macht Rosenblum sich auf eine innere Reise, beginnt aufzuräumen: »Ich lese viel, spreche mit Freunden, erledige wichtige Dinge. Eine friedliche Welt schaffen, damit ich sie schließlich klar und ruhig verlassen kann." (62)

Zunehmend erschöpft durch die fortschreitende Krankheit und die Strapazen der Chemotherapie wird Rosenblum im zweiten Jahr nach der Diagnose von Panikattacken überfallen und gerät in tiefe Trauer. Das Gefühl von Einsamkeit, unter dem sie seit ihrer Kindheit leidet, verstärkt sich. Es wird sie bis zu ihrem Tod begleiten. Dazwischen mischen sich auch Ärger und Wut: »Ich habe Frieden geschlossen mit Teilen meiner selbst, habe Teilen vergeben, bin anderen Teilen gegenüber nach wie vor verärgert. Ich bin wütend über eine unfaire, ungerechte, inkompetente und willkürliche Welt.» (81) Parallel dazu intensiviert sich auch der Trauerprozess Butlers, die schmerzlich miterlebt, wie sich ihre Lebenspartnerin mehr und mehr zurückzieht. Als Rosenblum gegen Ende des zweiten Jahrs beginnt, letzte Verfügungen zu treffen, wird Butler schockartig bewusst, dass die gemeinsame Zeit sich nun rasch dem Ende nähert: "Es war nun real. Sie musste sich auf ihren Tod vorbereiten. Sie würde sterben. Ich würde allein sein. Wir würden nicht gemeinsam alt werden.» (87). 
Barbara Rosenblums letztes Lebensjahr ist bestimmt durch strukturbildende Entscheidungen und Rituale: die Regelung des finanziellen Vermächtnisses, die Traufeier, die Mikwe und schließlich die Vorbereitungen für die Abdankungsfeier und das Begräbnis. Die von ihr getroffenen Entscheidungen bringen Klarheit und Entlastung. Die Auseinandersetzung mit der Fehldiagnose kommt zur Ruhe. Was sie sich bereits zu Beginn gewünscht hatte, ihr Leben noch ordnen zu können und neu zu sich zu finden, nimmt nun Gestalt an. Die Suche nach den passenden Formen für ihren »letzten Sabbat" gibt ihrer inneren Reise eine prospektive Ausrichtung. Ihre zunehmende Klarheit zeigt sich auch in dem Rundbrief, den sie ein Jahr vor ihrem Tod für ihren Freundeskreis schreibt und in dem sie zunächst die schlechte Botschaft mitteilen muss, dass der Krebs die Lunge befallen hat und ihre Lebenszeit vermutlich in sechs bis zwölf Monaten zu Ende gehen wird. Danach wendet sie ihre Aufmerksamkeit von der Krankheit weg und dem Leben zu:

"Ich habe den Eindruck, einfacher und einfacher zu werden, immer klarer zu sehen, was meine Essenz ist. Die Dinge sind nicht mehr so kompliziert. [...] Ich erfahre meine Entscheidungen und Präferenzen eher als einen Schritt Richtung Klarheit denn als Begrenzung. Mag man diesen Prozess auch Simplifizierung nennen. Ich erlebe es eher als ein Ankommen im eigenen vollsten und wahrsten Selbst und der schlichten Anerkennung dessen, was ist. [...] Ein Ankommen bei den wesentlichen Dingen. Ich nehme auch wahr, wie ich in eine neue Offenheit finde, eine Leichtigkeit, eine Ungezwungenheit, mit der ich derVollendung meines Lebens entgegenschaue. [...] Das Leben zittert mit Vibrationen des Lebendigen. Die Welt ist, wie es Gerard Manley Hopkins formulierte, 'gänzlich erfüllt mit der Herrlichkeit Gottesর. Ich schaue, wie Dinge zu sich selbst kommen, Augenblicke geboren werden, Dinge ihre Essenz offenbaren, Dinge im Übergang sind. Alle Dinge scheinen erfüllt mit einer Lebenskraft zu sein, einer Vitalität. Das Leben ist von seiner Lebendigkeit durchpulst und durchzittert. Das Leben ist geheimnisvoll, erfüllt, es bewegt sich in einer langsamen Fortbewegung wie eine Welle des Ozeans mit seinen unsichtbaren Tiefenströmungen." (105)

Obwohl sich das Gefühl der Verbundenheit in den letzten Lebensmonaten Rosenblums verdichtet, kämpft sie weiter mit ihrem Gefühl der Einsamkeit. Drei Wochen vor ihrem Tod schreibt sie: 
»Ich bin am Sterben. Ich werde bald sterben. Es bleibt mir ungefähr ein Monat. Und wenn ich die nächsten zwei Wochen noch schreiben kann, bin ich dankbar dafü. Verdammt noch mal! Bis zum allerletzten Tag! Das Schreiben ist mindestens so tief und zellartig wie der Krebs. [...] Alleine, ruhig, ich schaffe es nicht, dieses intensive Gefühl von Einsamkeit und Furcht zu vertreiben. Narkotika helfen. Sie lullen alles ein wenig ein, doch das tragische Zentrum meines Gehirns wird von Pillen kaum berührt. Deshalb: zwei kühle Martinis und eine Runde Weinen.« (162)

Rosenblum beginnt, sich ihr Sterben vorzustellen, wünscht sich ein sanftes, von Liebe getragenes Ende. Ihren letzten Tagebucheintrag schreibt sie an ihrem letzten Geburtstag. Mittels imaginierter Fotografien ihrer selbst vergegenwärtigt sie sich nochmals ihr nun beinahe abgeschlossenes Leben. Am Tag nach ihrem Tod beschreibt Sandra Butler die letzte gemeinsame Nacht, die von Zärtlichkeit und Trauer erfüllt ist, und führt dann die Lebenserzählung zu Ende

"Vor drei Tagen lagst du in deinem Bett, geschwollen von Ödemen, und schautest auf die Ärztin. Sie haben maximal noch wenige Wochenı, sagte diese, was ein unerwartetes Geschenk dieser brüsken Frau bedeutete. Am nächsten Tag schwollst du auf, dein aufgedunsener Körper konnte sich nicht mehr wenden, bewegen, gehen. Der wunde Mund macht das Sprechen unangenehm.Von Sätzen auf Worte reduziert, von Gläsern mit eisgekühltem Wasser zu einer Babyflasche - deine Hand war zu schwach, um ein Glas zu halten. IIch denke, es ist Zeit`, flüstertest Du mir zu. [...] Einmal mehr, wie schon so häufig im vergangenen Jahr, flüsterte ich, dass ich an deiner Seite sein würde, wenn der Tod käme. [...] Um genau Viertel nach $11 \mathrm{Uhr}$ am nächsten Morgen, dem Valentinstag, hast du mich in die Küche geschickt, um frisches Wasser zu holen, mir liebevoll zulächelnd. Als ich dein Zimmer wieder betrat, einige Augenblicke später, mit Kräutertee und eisgekühltem Wasser, warst du bereits tot. Deine Augen offen, dein Körper offengelegt, deine Lippen sanft geöffnet." (170)

\section{Würdigung}

Mit seltener Genauigkeit, die sich der Doppelperspektive und der erzählerischen Intensität und Direktheit der beiden Autorinnen verdankt, beschreibt Cancer in two voices die Wechselfalle und Phasen zweier Lebensgeschichten in zunehmender Todesnähe. In einer 
eigentümlichen Synchronisierung von erzählter Zeit und Lesezeit wird das Verrinnen der Lebenszeit lesend nacherlebbar. Die zwischen die Tagebuchnotizen eingeschobenen Reflexionen gleichen Atempausen, die das Zerrinnen der Tage unterbrechen. Die vier Storylines, die sich mehrfach verflechten, ohne ineinander aufzugehen, bilden einen dichten Klangteppich, vor dem sich die beiden Einzelstimmen der Erzählerinnen abheben. Dass das faktuale Erzählen einer Krankheits- und Sterbegeschichte, das den Widersinn von medizinischem Versagen, Krankheit und Tod sowie die Härte von Abschied und Trauer schonungslos zur Sprache bringt, dennoch so poetisch sein kann, ist die vielleicht grösste Überraschung, die das Buch bietet. Durch den jüdischen Hintergrund der beiden Autorinnen wird die erzählte Geschichte ihres gemeinsamen Schicksals in eine größere Geschichte eingeschrieben. So betrachtet ist das Buch auch ein zeitgeschichtliches Zeugnis, das die Lebensgeschichten zweier Frauen erzählt, die in den Jahren der nationalsozialistischen Judenverfolgung in unterschiedlichen jüdischen Milieus der USA geboren wurden und ihre persönliche und politische Identität wesentlich den Bewegungen der 1960er Jahre verdankten. In der Zwischenreflexion, die ihre Teilnahme an der Gedenkfeier zum 44. Jahrestags des Warschauer Aufstands beschreibt und die Überschrift Zeugnis ablegen trägt, überkreuzt sich das persönliche und das kollektive Schicksal, verbindet sich die persönliche Trauer mit jener einer ganzen Generation Überlebender. Am Ende ist Cancer in two voices die Geschichte einer Überlebenden. Das Buch endet mit einer kurzen Notiz Sandra Butlers zum 3. Jahrestag des Todes ihrer Lebenspartnerin. Auf einem Spaziergang winkt Butler einer alten Frau zu, die an einem Fenster eines Rehabilitationszentrums steht. Als diese zu lächeln beginnt und ihr zurückwinkt, bricht Butler unvermittelt in Tränen aus. Diese gelten den "Verlusten, die mein Herz belasten und der Entschiedenheit, die mir ermöglicht, durchzuhalten. In Liebe.«(183)

- Simon Peng-Keller ist Professor für Spiritual Care an der Theologischen und Medizinischen Fakultät der Universität Zürich und war 2013-2015 wissenschaftlicher Koordinator des NFP 67-Projektes »Hermeneutik des Vertrauens am Lebensende. Imaginatives Erleben und symbolische Kommunikation in Todesnähe«. 